\title{
An evolutionary band-limited Gaussian noise jamming algorithm for LMS-based GPS
}

\author{
Tao $\mathrm{Wu}^{*}$, Qiubai Zou, Jixiang $\mathrm{Wu}$, Wenqiang Li, and Zhenze Jia \\ Air Force Communication NCO Academy, China
}

\begin{abstract}
In this paper, evolutionary algorithm is used to optimize the flicker function of band-limited Gaussian noise interference, which disturbs the convergence of adaptive algorithm based on LMS in GPS receiver, so that the average null depth of GPS receiver under equal power is lower, and better jamming effect is achieved. The simulation results show that the jamming algorithm in this paper has better jamming effect than the band-limited Gaussian noise jamming in different jamming quantity.
\end{abstract}

\section{Introduction}

The traditional suppressed GPS jammer can not decode the GPS receiver correctly by transmitting jamming signal with higher power than GPS signal. The antenna array is used in the nulling GPS receiver, and the interference direction is nullified by the adaptive algorithm, which has significant anti-jamming effect on the traditional suppression jamming mode. Most researchers still study from the angle of jamming ordinary GPS receiver. Reference [1-2] respectively proposed the best parameter selection strategy and the method of judging the best jamming signal. A new wideband comb spectrum jamming method is proposed in reference [3]. Reference [4] analysed the influence of continuous wave interference on GPS receiver acquisition. There is little research on the interference of nulling GPS. In reference [5], a pulse jamming method for GPS nulling is proposed. The relative delay between multiple interferences is adjusted periodically to obtain a certain performance gain. The influence of band-limited Gaussian noise on different bandwidth is evaluated in reference [6]. Reference [7] gives the optimal interference power difference of GPS receiver for fixed step LMS.

For GPS receiver based on LMS, this paper proposes an interference algorithm which optimizes the jamming scintillation function by evolutionary algorithm. Compared with the band-limited Gaussian noise, the proposed algorithm has a significant performance gain when the number of interferences is large. Section 2 introduces the model of LMS-based GPS receiver. Section 3 introduces the jamming algorithm in this paper. In Section 4, the performance of the algorithm is verified by simulation experiments. Section 5 summarizes the algorithm.

\footnotetext{
* Corresponding author: wutao996@126.com
} 


\section{LMS-based GPS receiver model}

The structure of LMS-based GPS receiver model is shown in Fig. 1. The signals received by $M$ antenna array elements are obtained by the adaptive algorithm based on LMS to obtain the optimal weight $W$. The signals weighted by the optimal weight $W$ are demodulated by the GPS receiver to obtain the GPS information. The optimal weight W can be used to calculate the pattern $G$. The direction nulling depth of interference signal in the pattern is far greater than that in other directions. The influence of interference signal on the receiver can be evaluated by the null depth of the pattern.

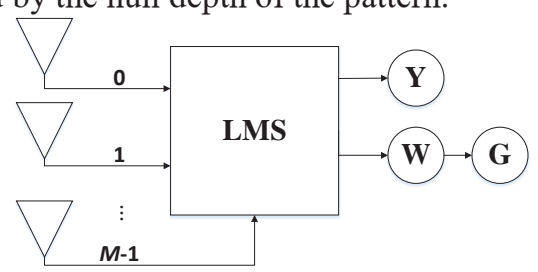

Fig. 1. LMS-based GPS receiver model.

\subsection{Receiving antenna array signal}

The uniform circular array is often used for LMS-based GPS antenna array. Fig. 2 shows a uniform circular array with seven elements. The circular array element is selected as the reference array element, and the received signal expression of the reference array element is assumed to be:

$$
x_{0}(t)=A(t) e^{j(\omega t+\varphi)}
$$

where $A(t)$ is the signal amplitude and $\varphi$ is the initial phase.

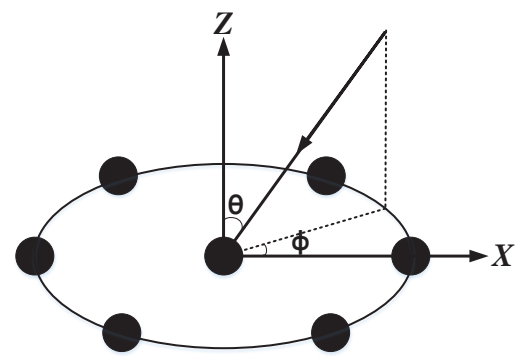

Fig. 2. Uniform circular array with seven elements.

The $M$ array elements without reference are expressed as $\left[x_{1}(\mathrm{t}) x_{2}(\mathrm{t}) \ldots x_{M-1}(t)\right]^{T}$, and the propagation delay with the reference element is $\left[\tau_{1}, \tau_{2}, \ldots, \tau_{M-1}\right]$. Considering that for narrow-band signal $A(t-\tau) \approx A(t)$, the received signal of array element can be expressed as:

$$
X=A x_{0}(t)
$$

where $X=\left[x_{0}(\mathrm{t}) x_{1}(\mathrm{t}) x_{2}(\mathrm{t}) \ldots x_{M-1}(t)\right]^{T}$ and $A=\left[1 e^{-j \omega \tau_{1}} e^{-j \omega \tau_{2}} \ldots e^{-j \omega \tau_{M-1}}\right]^{T}$.

In the above formula, $A$ is called the guidance vector. For a uniform circular array with seven elements, if the radius of the element is taken as half wavelength, i.e. $r=\lambda / 2$, the guidance vector can be obtained as follows: 


$$
A(\theta, \varphi)=\left[\begin{array}{c}
1 \\
e^{-j \pi \sin \theta \cos \varphi} \\
e^{-j \pi \sin \theta \cos (\varphi-\pi / 3)} \\
e^{-j \pi \sin \theta \cos (\varphi-2 \pi / 3)} \\
e^{-j \pi \sin \theta \cos (\varphi-\pi)} \\
e^{-j \pi \sin \theta \cos (\varphi-4 \pi / 3)} \\
e^{-j \pi \sin \theta \cos (\varphi-5 \pi / 3)}
\end{array}\right]
$$

where $\theta$ is the signal pitch angle and $\varphi$ is the signal azimuth angle.

It is assumed that the received signal $R(t)$ of the array element is contained of $N_{S}$ GPS signals, $N_{J}$ jamming signals and additive white Gaussian noise. Then the received signal $R$ of the array element is expressed as:

$$
R(t)=\sum_{i=1}^{N_{S}} A_{S_{i}} S_{i}(\mathrm{t})+\sum_{j=1}^{N_{J}} A_{j} J_{j}(\mathrm{t})+N(t)
$$

where $A_{S_{i}}$ refers to the guidance vector of the $i$ th GPS signal, $A_{j}$ is the guidance vector of the $j$ th jamming signal, $S_{i}(t)$ represents the received signal of the ith GPS signal at the reference array element, $J_{j}(\mathrm{t})$ represents the received signal of the $j$ th jamming signal at the reference array element, and $N(\mathrm{t})$ represents additive white Gaussian noise.

\subsection{LMS and pattern}

LMS algorithm minimizes the mean square error between the filtered output signal and the expected signal. In LMS-based GPS, the output signal of reference array element is regarded as the expected signal $d(k)$, that is, $d(k)=x_{0}(k)$. The weighted average $y(k)$ of the other array elements is used as the filtered output signal:

$$
y(k)=W^{T} X(k)
$$

where $X(k)=\left[x_{1}(k), x_{2}(k), \ldots, x_{M-1}(k)\right]^{T}$ and $W=\left[w_{1}, w_{2}, \ldots, w_{M-1}\right]^{T}$.The error signal $e(k)=d(k)-y(k)$. LMS algorithm uses steepest descent method to get weight vector:

$$
W(k+1)=W(k)-\mu \nabla_{w} E\left\{|e(k)|^{2}\right\}=W(k)-2 \mu X(k) e(k)^{T}
$$

where $\mu$ is the iteration step size. The optimal weight is obtained by LMS algorithm as $W_{\text {opt }}=\left[\begin{array}{llll}W_{1} & W_{2} & \ldots & W_{M-1}\end{array}\right]^{T}$, then the weight of each element can be expressed as:

$$
W=\left[1,-W_{1},-W_{2}, \ldots,-W_{M-1}\right]^{T}
$$

Then the pattern is expressed as:

$$
G(\theta, \varphi)=W^{T} A(\theta, \varphi)
$$

\section{Evolutionary band-limited Gaussian noise jamming algorithm}

The complex domain expression of jamming signal is as follows:

$$
x(n T)=r(n T)\left[f_{1}(n T) \cos \left(2 \pi f_{c} n T+\beta\right)+j f_{2}(n T) \sin \left(2 \pi f_{c} n T+\beta\right)\right]
$$

where $f_{c}$ is carrier frequency, $\beta$ is initial phase, $T=1 / f_{s}$ is the sampling period. $f_{1}(n T)$ and $f_{2}(n T)$ denotes the Gaussian white noise after $H(z)$ filtering, and its $z$-domain expression is $H(z)=1 /\left(1-0.9 z^{-1}\right) \cdot r(n T)$ denotes the scintillation function as follows:

$$
r(n T)=\left\{\begin{array}{cc}
1 & n \epsilon\left(F_{a}(2 k), F_{a}(2 k+1)\right], k=0,1,2, \ldots \\
0 & \text { other }
\end{array}\right.
$$

where $F_{a}(k)=\sum_{i=1}^{k} a(k)$ and $F_{a}(0)=0$.

The evolutionary band-limited Gaussian noise jamming algorithm optimizes the $a(k)$ sequence by evolutionary algorithm, and then optimizes the scintillation function, so that the GPS receiver can get smaller null in the interference direction. The algorithm is as follows: 
Initialization signal parameter: $f_{c}, f_{S}, N_{S}, N_{J}, \theta, \varphi$. Initialization the parameters of evolutionary algorithm, such as and the number of population members $N$, the maximum number of iterations $N_{\text {loop }}$, cross rate $\alpha$, etc.

The following steps cycle $N_{\text {loop }}$ times:

Step 1: calculate the variogram $a(k)=\operatorname{Mutate}(a(k))$.

Step 2: calculate the crossover function $a(k)=\operatorname{Cross}(a(k), \alpha)$.

Step 3: calculate the evaluation value of each member scores $=\operatorname{Score}(a(k))$.

Step 4: record the maximum evaluation value scores $=\operatorname{Score}(a(k))$.

Among them, the Mutate function takes the random value in the range of $a(k)$ random position. Cross exchanges the members of $\alpha N$ and exchanges half of the data randomly. The Score function first calculates the jamming signal, and then calculates the optimal weight $W_{o p t}$ by LMS algorithm, then the null depth of the pattern $G(\theta, \varphi)$ in the direction of the interference signal is returned.

\section{Simulation experiment}

In this section, we compare the performance difference between evolutionary band-limited Gaussian noise jamming algorithm and traditional band-limited Gaussian noise jamming algorithm through simulation experiments. The number of interference signals in the experiment of $N_{J}$ is $1 \sim 6$ for testing, and the average value of 100 groups of sample data is taken as the average null depth. The other parameters are: the number of array elements $M$ $=7$; the radius of antenna array $r=\lambda / 2$; and the sampling rate $f_{s}=3.2 \mathrm{GHz}$; the length of single sample data is 10000 ; the number of GPS signals is $N_{s}=1$; the elevation angle of GPS signal is $70^{\circ}$ and the azimuth angle is $10^{\circ}$; the elevation angle of jamming signal is $70^{\circ}$ and the azimuth angle is $40^{\circ}, 70^{\circ}, 100^{\circ}, 150^{\circ}, 250^{\circ}$ and $320^{\circ}$ respectively; the signal-tonoise ratio $\mathrm{S} / \mathrm{N}=-30 \mathrm{~dB}$; the jamming noise ratio $\mathrm{J} / \mathrm{N}=30 \mathrm{~dB}$. The experimental results are shown in Table 1.

Table 1. Comparison of null depth between band-limited Gaussian noise jamming algorithm and evolutionary band-limited Gaussian noise jamming algorithm.

\begin{tabular}{|c|c|c|c|}
\hline $\begin{array}{c}\text { Interference } \\
\text { number } N_{J}\end{array}$ & $\begin{array}{c}\text { Band-limited } \\
\text { Gaussian noise }\end{array}$ & $\begin{array}{c}\text { Evolutionary } \\
\text { algorithm }\end{array}$ & $\begin{array}{c}\text { Gain of } \\
\text { performance }\end{array}$ \\
\hline 1 & $48.85 \mathrm{~dB}$ & $43.14 \mathrm{~dB}$ & $5.71 \mathrm{~dB}$ \\
\hline 2 & $48.66 \mathrm{~dB}$ & $42.97 \mathrm{~dB}$ & $5.69 \mathrm{~dB}$ \\
\hline 3 & $50.11 \mathrm{~dB}$ & $44.52 \mathrm{~dB}$ & $5.59 \mathrm{~dB}$ \\
\hline 4 & $47.60 \mathrm{~dB}$ & $41.94 \mathrm{~dB}$ & $5.66 \mathrm{~dB}$ \\
\hline 5 & $41.59 \mathrm{~dB}$ & $32.83 \mathrm{~dB}$ & $8.76 \mathrm{~dB}$ \\
\hline 6 & $29.81 \mathrm{~dB}$ & $20.04 \mathrm{~dB}$ & $9.77 \mathrm{~dB}$ \\
\hline
\end{tabular}

It can be seen from table 1 that the null depth of traditional band-limited Gaussian noise algorithm is basically stable in the interference direction when the number of interference is $1 \sim 4$, and it is significantly improved when the number of interference is $5 \sim 6$. The results show that the null depth of evolutionary band-limited Gaussian noise jamming algorithm has similar conclusion when the number of interferences is different. Compared with the traditional band-limited Gaussian noise jamming algorithm, the performance gain of evolutionary band-limited Gaussian noise jamming algorithm is about $5 \sim 9 \mathrm{~dB}$, and the performance difference increases with the increase of interference quantity. 


\section{Summary}

This paper describes the GPS receiver based on LMS, and proposes an interference algorithm through optimize flicker function by evolutionary algorithm. The performance of the algorithm is analyzed by interference null depth of pattern. The algorithm can disturb the convergence of LMS better by optimizing the flicker function, and obtain better performance gain. The performance of the algorithm is significantly better than that of band limited Gaussian noise interference algorithm.

\section{References}

1. Mao Hu, Wu Dewei. Analysis of Jamming Parameters Selecting of Band-limited Gaussian Noise to GPS Military Signals[J]. Journal of air force engineering university(natural science edition), 15(6), 58-62 (2014)

2. Wang Jiao, Su Zhong, Zhang Yuexia. Study on Optimal Jamming Signal of GPS System[J]. Computer Measurement \& Control, 24(4), 257-267 (2016)

3. Mao Hu, Wu Dewei, Lu Hu, Yan Zhanjie. Analysis of a New Wideband Blanket Jamming Type to GPS Receiver [J]. Journal of Electronics \& Information Technology, 12(36), 2929-2933 (2014)

4. Ye, F., H. Tian, and F. Che. CW interference effects on the performance of GPS receivers[C]. 2017 Progress In Electromagnetics Research Symposium - Fall (PIERS FALL), 66-72 (2017)

5. Li Yawei. Research on Jamming and Anti-jamming about Satellite Navigation Receiver with Array Antenna[D]. Xidian University (2017)

6. Betz J W, Kolodziejski K R . Generalized Theory of Code Tracking with an Early-Late Discriminator Part II: Noncoherent Processing and Numerical Results[J]. IEEE Transactions on Aerospace \& Electronic Systems, 45(4), 1538-1556 (2009)

7. Liu F, Wang $\mathrm{Y}$, Yue L, et al. JAMMING METHOD BASED ON OPTIMAL POWER DIFFERENCE FOR LMS-GPS RECEIVER[J]. Progress in Electromagnetics Research M, 77, 167-175 (2019)

8. Liu F, Du R , Bai X . A virtual space-time adaptive beamforming method for spacetime antijamming[J]. Progress in Electromagnetics Research M, 58, 183-191 (2017)

9. Turan C. A new variable step-size block LMS algorithm for a non-stationary sparse systems[C] Twelve International Conference on Electronics Computer \& Computation. IEEE (2016) 